\title{
Patterns of pediatric thoracic penetrating injuries
}

\author{
A single-trauma-center experience in Riyadh, Saudi Arabia
}

Suliaman M. Alaqeel, MBBS, Abdulaziz A. Howsawi, MBBS,

Mohammad K. Al Namshan, MBBS, MPH, Jamila O. Al Maary, MBBS.

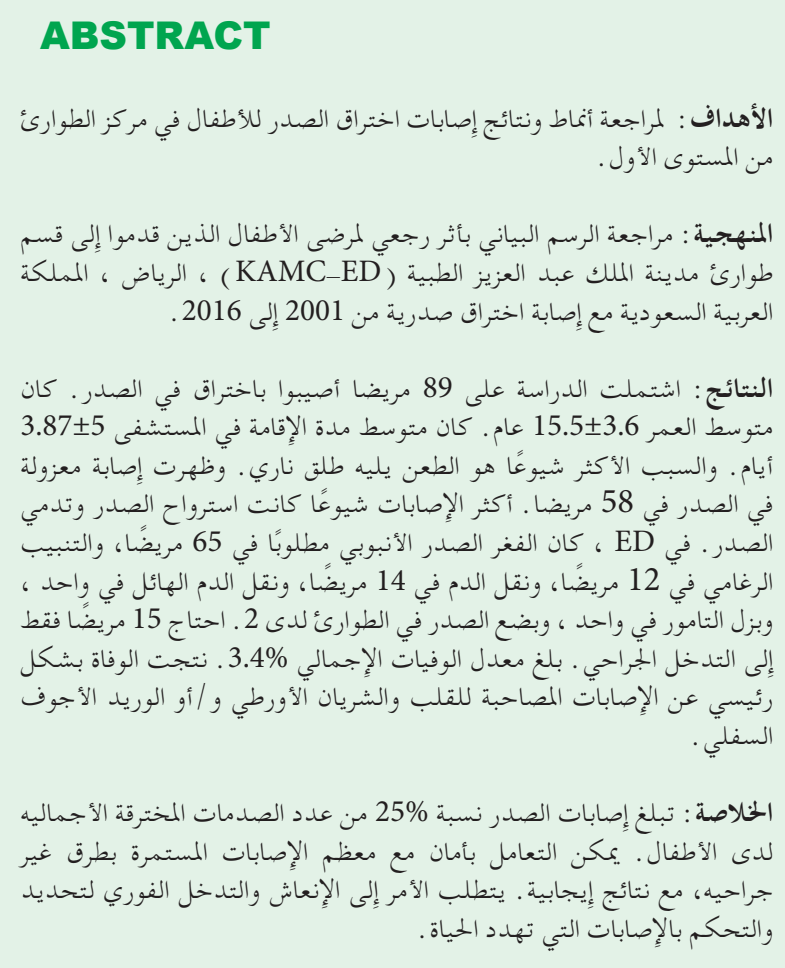

Objectives: To review the patterns and outcomes of pediatric thoracic penetrating injuries in a level one trauma center.

Methods: Retrospective chart review of pediatric patients who presented to the King Abdulaziz Medical City Emergency Department (KAMC-ED), Riyadh, Saudi Arabia with thoracic penetrating injury from 2001 to 2016.

Results: Eighty-nine patients had a penetrating injury to the thorax were identified. The mean age was 15.5 \pm 3.6 years. The mean length of hospital stay was $3.87 \pm 5$ days. The most common cause was stabbing followed by gunshot. Isolated injury to the thorax was seen in 58 patients. The most common injuries sustained were pneumothorax and hemothorax. In the $\mathrm{ED}$, tube thoracostomy was required in 65 patients, endotracheal intubation in 12, blood transfusion in 14, massive blood transfusion in one, pericardiocentesis in one, and ED thoracotomy in 2. Only 15 patients required surgical intervention. The overall mortality rate was $3.4 \%$. Death was mainly caused by associated injuries to the heart, aorta and/ or inferior vena cava.

Conclusion: Thoracic injuries represent $25 \%$ of the overall penetrating traumas in pediatric age group. Most sustained injuries can be safely managed nonoperatively, with a favorable outcome. Prompt resuscitation and intervention are required to identify and manage life-threatening injuries.

Keywords: penetrating injury, thorax, pediatric, outcome

Saudi Med J 2021; Vol. 42 (3): 280-283 doi: 10.15537/smj.2021.42.3.20200693

From the Department of Pediatric Surgery (Alaqeel, Al Namshan, Al Maary), King Abdullah Specialized Children's Hospital, King Abdulaziz Medical City and from the Department of Family Medicine (Howsawi), Prince Sultan Military Medical City, Riyadh, Kingdom of Saudi Arabia.

Received 1st November 2020. Accepted 14th January 2021.

Address correspondence and reprint request to: Dr. Suliaman $M$. Alaqeel, Department of Pediatric Surgery, King Abdullah Specialized Children's Hospital, King Abdulaziz Medical City, Riyadh, Kingdom of Saudi Arabia.E-mail:sm.alaqeel@gmail.com

ORCID ID: https://orcid.org/0000-0003-2778-9710

Disclosure. The authors declare no conflicts of interest associated with this publication. Also, no financial support or fund for this work from drug companies, private or governmental institutions that could have influenced its outcome. 
$\mathrm{T}$ rauma is responsible for $9 \%$ of the overall mortality in all age groups. ${ }^{1}$ Among children, the incidence of penetrating trauma varies from $10-22 \% .^{2-4}$ Few publications have addressed penetrating injury in pediatrics, particularly injury to the thorax. The estimated mortality risk from penetrating trauma is high and ranges from $4 \%$ to $25 \% .^{5}$ Due to lack of local data, the aim of this study is to share our experience and review the patterns, characteristics and outcomes of pediatric thoracic penetrating injuries in a single level one trauma center in Saudi Arabia.

Methods. A study proposal was submitted to King Abdullah International Medical Research Center (KAIMRC), Riyadh, Saudi Arabia and went through a process of institutional review board (IRB) assessment, research committee review and peer review. After approval, eligibility and accessibility to the trauma registry database become available. From the King Abdulaziz Medical City (KAMC) data, we retrieved the medical records of all patients who sustained penetrating injury over a 16-year study period.

All patients (18 years or younger) who presented to the KAMC emergency department (KAMC-ED) with penetrating trauma from January 2001 to December 2016 were included. Those who sustained penetrating injury to the thorax and were initially managed in KAMC-ED were selected. Any transferred cases from other institutions were excluded.

Medical charts were retrieved and reviewed by the coinvestigators. Data collected included patient demographics (age, sex), place of injury (home, school, street), mode of transportation to the hospital (private car, ambulance), duration from injury to ED, mechanism of injury (MOI), admission physiology (heart rate $[\mathrm{HR}]$, respiratory rate $[\mathrm{RR}]$, blood pressure $[\mathrm{BP}])$, type of injury, associated injuries, ED management, operative intervention, length of hospital stay, intensive care unit (ICU) stay and mortality rate.

Statistical analysis. We used IBM Statistical Package for Social Sciences for windows, Version 22.0 (IBM Corp., Armonk, NY, USA) for data analysis. Quantitative variables are presented as mean \pm standard deviation (SD), and qualitative data are presented as frequencies and percentages.

Results. A total of 355 patients were hospitalized for penetrating injuries over the study period. Eighty-nine patients sustained penetrating injury to the thorax. All patients were male except one female. Their mean age was $15.5 \pm 3.6$ years (1-18). The place of injury (home, school, street), mode of transportation (private car, ambulance), and duration from injury to ED are summarized in Table 1.

In the ED, initial hemodynamics upon arrival were retrieved. Normal values were adjusted according to patient age. Almost half of the presenting patients were stable and had normal hemodynamics upon arrival $(\mathrm{n}=49)$. The mean HR was $98 \pm 24$ beats $/ \mathrm{min}$, and the mean systolic blood pressure (SBP) was $121 \pm 25$ SD. Abnormal or unstable hemodynamics according to patients age, such as tachycardia (31.4\%), tachypnea $(72 \%)$ and hypotension $(5.6 \%)$ were recorded. Initial ED hemodynamics were not documented in 9 patients.

The mechanism of injury (MOI) was stabbing injury (SI) in $71(79.8 \%)$, gunshot injury (GSI) in $11(12.4 \%)$, air gun in $4(4.5 \%)$ and sharp objects such as glass in $3(3.3 \%)$. Isolated penetrating injury to the thorax was encountered in 58 patients, while 31 patients had associated injuries to other body regions $(65.2 \%$ versus $34.8 \%)$. The types of injuries sustained are summarized in Table 2.

Table 1 - Overview of pre-hospital data.

\begin{tabular}{lc}
\hline Pre-hospital data & $\mathbf{n}(\%)$ \\
\hline Place of injury & $3(3.4)$ \\
Home & $2(2.2)$ \\
School & $7(7.9)$ \\
Street & $77(86.5)$ \\
ND* & \\
\hline Mode of transportation & $21(23.6)$ \\
Ambulance & $67(75.3)$ \\
Private car & $1(1.1)$ \\
ND* & \\
\hline Duration from injury to ED & $39(43.8)$ \\
$<1$ hour & $7(7.9)$ \\
1-2 hours & $1(1.1)$ \\
2-6 hours & $42(47.2)$ \\
Not specified & \\
\hline \multicolumn{2}{l}{$*$ Not documented } \\
\hline
\end{tabular}

Table 2 - Type of injuries encountered in penetrating thoracic injuries.

\begin{tabular}{lc}
\hline Type of injury & $\mathbf{n}(\%)$ \\
\hline Pneumothorax & $63(70.7)$ \\
Hemothorax & $23(25.8)$ \\
Lung contusion/laceration & $7(7.8)$ \\
Diaphragm injury & $5(5.6)$ \\
Hemopericardium & $4(4.49)$ \\
Cardiac injury & $4(4.49)$ \\
Major vessel injury & $3(3.4)$ \\
Pneumomediastinum & $2(2.2)$ \\
Esophageal injury & $1(1.1)$ \\
Rib Fracture & $1(1.1)$ \\
Solid/visceral abdominal organ injury & $12(13.4)$ \\
\hline
\end{tabular}


Table 3 - List of surgical interventions needed in managing penetrating thoracic injuries.

\begin{tabular}{lc}
\hline Operative approach* & n (\%) \\
\hline Thoracotomy & $4(4.49)$ \\
Median sternotomy & $1(1.1)$ \\
Thoracoscopy & $2(2.2)$ \\
Laparotomy & $5(5.6)$ \\
Diagnostic Laparoscopy & $2(2.2)$ \\
Wound Exploration and closure & $1(1.1)$ \\
Bullet retrieval by IR* & $1(1.1)$ \\
\hline \multicolumn{2}{c}{ *Multiple approaches were necessary in one patient. } \\
\multicolumn{2}{c}{ *Intervention radiology } \\
\hline
\end{tabular}

In the $\mathrm{ED}$, tube thoracostomy was required in 65 patients $(73 \%)$, endotracheal intubation in $12(13.5 \%)$, blood transfusion in $14(15.7 \%)$, massive transfusion protocol in one $(1.1 \%)$, pericardiocentesis in one $(1.1 \%)$ and ED thoracotomy in 2 patients $(2.2 \%)$.

The majority of patients required conservative management, but operative intervention was required in $17 \%(\mathrm{n}=15)$. Different surgical approaches were needed, and in some patients, multiple approaches were necessary (Table 3). The main indications for surgery were massive hemothorax, lung laceration, cardiac injury, aortic injury, esophageal injury, diaphragmatic injury, pericardial hematoma and solid/visceral abdominal organ injury.

The overall outcome was favorable, and patients were discharged home in stable condition. The ICU admission was not required in $83(93 \%)$ patients, who were observed in regular wards. The mean length of hospital stay was $3.87 \pm 5$ days, and the average length of ICU stay was $6 \pm 6$ days. The mortality rate was $3.4 \%(n=3)$. Death was observed only in the GSI group. Three patients sustained injuries to the heart, aorta and/ or inferior vena cava. They arrived at the ED with a mean HR of 77 beats/min and a mean SBP of 63 . Heart rate and SBP were unrecordable in one. Their injury severity scores (ISSs) were 25, 25 and 41, respectively. None of them survived, and they died immediately after emergency thoracotomy.

Discussion. The present study retrospectively reviewed the patterns and outcomes of penetrating thoracic injuries among children in our region. The study was conducted in KAMC, which is a level one trauma center located in eastern Riyadh. It covers a large area with a high crime rate and low socioeconomic status. The hospital ED is reachable, is easily accessible and provides around-the-clock trauma service.
Prehospital evaluation and assessment can predict the outcome of trauma patients. ${ }^{6,7}$ Patients typically arrive at our ED without prior notification. Most of them arrive by their own vehicles. The time from injury to ED was not specified in $47.2 \%(n=42)$ of our cases. However, some patients reached the hospital within one hour (or less) and received immediate definite care $(n=39)$. It is not known whether these confounding factors affected the outcome, but it has been reported that private vehicle transport significantly reduced in-hospital mortality in this subset of patients. ${ }^{6}$ Our prehospital service is not well established, and data including hemodynamics at the scene and during transport and resuscitation at the scene and during transport were lacking. Moreover, the mortality rate of those who could never reach the hospital and died at the scene is missing.

Due to governmental restrictions on firearm possession, stabbing injury (SI) by far predominated in our region. Numerous studies have had similar results, where gunshot injuries (GSIs) represented the second most common MOI after stabbing., ${ }^{5,9}$ When we compared the SI and GSI groups, we found significant differences between the 2 groups in the clinical presentation, management, and need for surgical intervention. ${ }^{10}$ The mean ISS was $8 \pm 3$ in the SI group compared to $14 \pm 12$ in the GSI group. The SI group required tube thoracostomy more often than surgery ( $83 \%$ vs. $7 \%)$. In contrast, the GSI group was unstable upon arrival and required immediate surgical intervention. Mortality was reported in the GSI group (3 of 11), but not in the SI group.

Two-thirds of patients had a penetrating injury isolated to the thorax (65.2\%). Pneumothorax was the most common injury encountered and was managed by tube thoracostomy. Surgical intervention was required in $17 \%$ of patients and was indicated secondary to lung laceration, massive hemothorax, or suspected or confirmed diaphragmatic injury. The surgical intervention in other pediatrics studies was needed in $33 \%$ to $45 \%$ of the cases. ${ }^{2,5}$ Some patients with pneumothorax or hemothorax required laparotomy for other associated injuries. Associated solid/visceral organ injury was seen in the liver in 4 cases, stomach in 3, large bowel in 3, and spleen, kidney and small bowel in one each. Eight patients who sustained injury to the diaphragm were found to have concomitant abdominal organ injuries. ${ }^{13}$

This study demonstrated an acceptable overall outcome of penetrating thoracic injury among children. The majority of patients were discharged home in stable condition and did not require any ICU admission. This 
study had a mortality rate of $3.4 \%$ while in similar other studies in children the reported mortality rate was $4 \%-25 \% .^{5}$ In our review, a subset of patients who sustained penetrating injury to the heart, pericardium, or major thoracic vessels did not survive. In this subset, the type of injury sustained (GSI), unstable hemodynamics upon arrival, high ISS score (average 30) and ED thoracotomy were factors that could predict their outcome from the start. ${ }^{5}$ Studies that have addressed the role of ED thoracotomy in pediatric penetrating thoracic trauma have revealed no clear evidence supporting its utilization in resuscitation. .,11,12 $^{2}$

Study limitations. First, it is a retrospective single center study. Second, pre-hospital data were missing and data related to pre-hospital management could not be retrieved. Also, long-term follow up of the patients were not available in all patients. Nevertheless, this the first study, to our knowledge, that focused on penetrating thoracic injuries in pediatric age group in our region.

In conclusion, thoracic penetrating injuries represent $25 \%$ of the overall penetrating traumas in the pediatric age group in Riyadh. The mortality rate was 3.4\%. Mortality rate in this study can be attributed to multiple factors, including a predominance of low-velocity stabbing injury among our cases. Another reason is that most sustained injuries were injury to the pleura and lung parenchyma; these injuries were safely managed non-operatively. Last, patients who sustained major vessel and/or cardiac injury represented the minority but carried fatal outcome. Further research is required to focus on the causes and preventive measures of such injuries.

\section{References}

1. World Health Organization. Injuries and violence: the facts 2014. [Updated 2014; date accessed: ????]. Available from URL: https://www.who.int/violence_injury_prevention/media/ news/2015/Injury_violence_facts_2014/en/\#:-:text=A\%20 n ew $\% 20$ d o cum en t \% 20 by $\% 20$ W H O, and $\% 20$ poisonings $\% 2 \mathrm{C} \% 20$ among $\% 20$ other $\% 20$ causes.
2. Nance M, Sing R, Reilly P, Templeton J, Schwab C. Thoracic gunshot wounds in children under 17 years of age. J Pediatr Surg 1996; 31: 931-935.

3. Moore H, Moore E, Bensard D. Pediatric emergency department thoracotomy: A 40-year review. J Pediatr Surg 2016; 51:315-318.

4. Allen C, Valle E, Thorson C, Hogan A, Perez E, Namias N et al. Pediatric emergency department thoracotomy: A large case series and systematic review. J Pediatr Surg 2015; 50: 177-181.

5. Mollberg N, Tabachnick D, Lin F, Merlotti G, Varghese T, Arensman $\mathrm{R}$ et al. Age-associated impact on presentation and outcome for penetrating thoracic trauma in the adult and pediatric patient populations. J Trauma Acute Care Surg 2014; 76: 273-278.

6. Boleken M, Cevik M, Yagiz B, Ter M, Dorterler M, Aksoy T. The characteristics and outcomes of penetrating thoracic and abdominal trauma among children. Pediatr Surg Int 2013; 29: 795-800.

7. Ceran S. Chest trauma in children. Eur J Cardiothorac Surg 2002; 21: 57-59.

8. Wandling M, Nathens A, Shapiro M, Haut E. Association of Prehospital Mode of Transport With Mortality in Penetrating Trauma. JAMA Surg 2018; 153: 107.

9. Moskowitz E, Burlew C, Kulungowski A, Bensard D. Survival after emergency department thoracotomy in the pediatric trauma population: a review of published data. Pediatr Surg Int 2018; 34: 857-860.

10. Fitch J, Dieffenbaugher S, McNutt M, Miller C, Wainwright D, Villarreal J, et al. Are We out of the woods yet? The aftermath of resuscitative thoracotomy. J Surg Res 2020; 245: 593-599.

11. Segalini E, Di Donato L, Birindelli A, Piccinini A, Casati A, Coniglio $\mathrm{C}$ et al. Outcomes and indications for emergency thoracotomy after adoption of a more liberal policy in a western European level 1 trauma centre: 8-year experience. Updates Surg 2018; 71: 121-127.

12. Heus C, Mellema J, Giannakopoulos G, Zuidema W. Outcome of penetrating chest injuries in an urban level I trauma center in the Netherlands. Eur J Trauma Emerg Surg 2015; 45: 461-465.

13. Onat S, Ulku R, Avci A, Ates G, Ozcelik C. Urgent thoracotomy for penetrating chest trauma: Analysis of 158 patients of a single center. Injury 2011; 42: 900-904. 